\title{
Mixed Expectations in the Recruitment Settlement during COVID-19 Pandemic: Experiences of Newly Employed Nurses in Oman
}

\author{
Mudhar Al-Adawi', Samiha Al-Habsi' ${ }^{1}$, Nasra Al-Hashmi1 ${ }^{1}$, Salma Al-Bulushi ${ }^{1}$, Kamila Al-Alawi ${ }^{*}$ \\ ${ }^{1}$ Royal Hospital, Ministry of Health (MOH), Muscat, Oman \\ ${ }^{2}$ WHO-Country Office Oman, Muscat, Oman \\ Email: ^alalawikamila@gmail.com
}

How to cite this paper: Al-Adawi, M., Al-Habsi, S., Al-Hashmi, N., Al-Bulushi, S., and Al-Alawi, K. (2021) Mixed Expectations in the Recruitment Settlement during COVID-19 Pandemic: Experiences of Newly Employed Nurses in Oman. Open Journal of Nursing, 11, 542-556. https://doi.org/10.4236/ojn.2021.116046

Received: May 24, 2021

Accepted: June 27, 2021

Published: June 30, 2021

Copyright $\odot 2021$ by author(s) and Scientific Research Publishing Inc. This work is licensed under the Creative Commons Attribution International License (CC BY 4.0).

http://creativecommons.org/licenses/by/4.0/ (c) (i) Open Access

\begin{abstract}
Introduction: Hospital nurses' recruitment during the COVID-19 pandemic is a major challenge for the hospital, the staff themselves and for their families \& relatives. Although the world has undergone different pandemics and disasters, the literature has shown that the new nurses still face uncertainty in dealing with health emergencies and disasters that impose significant stress on their performance at work. The situation in Oman is not different and little is known about the newly employed nurses' experiences during the COVID-19 pandemic. Method: The qualitative study was designed to include face to face interviews of the newly employed nurses between May and December 2020 at the biggest tertiary hospital in Muscat, the capital of Oman to explore their experiences and how they confronted the COVID-19 pandemic challenges during their internship period. Result: The data was analyzed through qualitative manifest content analysis and disclosed different experiences which were framed into four contexts: Introductory Nurses Plan, Hospital Environment, Nurses Characters and Nurses Families \& Relatives. The negative experiences were mainly related to the intense internship requirements, non-availability of the preceptors, hospital environment and COVID-19 circumstances. On the other hand, the positive experiences were mainly related to their self-motivation, recruitment satisfaction, and family encouragement and support. Conclusion: Clear nurses' lack of knowledge towards COVID-19 pandemic was disclosed and associated with mixed emotional insights towards themselves, their families and relatives, their preceptors and towards the hospital environment including their preparatory plan. The study identified the nurses' stressors and motivators that could be ad-
\end{abstract}


dressed with a clear need for preparatory adjustments to support newly employed nurses in future appointments with a lot of involvements shared responsibilities and implications.

\section{Keywords}

COVID-19 Pandemic, Experiences, Newly Employed Nurses, Oman

\section{Introduction}

Globally, Corona Viruses (CoVs) are defined as a group of highly diverse, enveloped single-stranded RNA viruses [1].

Oman has been affected by (COVID-19) in February 2020 with the first two reported cases from Iran as Omani residents travelled abroad and came back to their home country [2] [3]. Accordingly, the cases started to rise and the government started to apply community measures which led eventually to the semi-closure of many cities in the country. The fundamental problem at that time was associated with a dramatic increase in diagnosed cases in a homogenous population due to socio-economic reasons [2] and due to a very unique lifestyle of the country [4]. As time progress, the pandemic of (COVID-19) continued to evolve, and since there is no predictable pattern for the virus and the duration of the vaccine immunity is not known yet, the World Health Organization (WHO) recommended certain measures to control the spread of disease.

Due to the disease ambiguity and the rapid changes in the international guidelines and strategies, the country demanded rapid measures' adaption, particularly among health care workers. Nurses were highlighted as frontline defenders who were not only absent but if available not prepared on how to manage the current crisis. Therefore, when hospitals started to be conquered with patients, the shortage of nurses was among the main challenges including their unpreparedness to confront the disease personally, at the hospital level, at the community level and nationally [5].

Accordingly, the government initiated a process to recruit additional new nurses (who finished their nursing degree and waiting for their employment) to the hospitals which are considered to be the (COVID-19) centers in the country. This strategy was established to cope with the continuous increase in the number of cases in the country and to develop a backup plan to handle the (COVID-19) pandemic.

According to the literature, the transition of the new nurses and their experiences in their first years of recruitment proven to have a significant influence on their career and it continues to affect their possibility of leaving their profession between the first and fifth year of professional practice [6]. Although several studies published earlier explored the transition of recently graduated nurses and their confidence level in different specialties [7] [8], little is known on how these graduates are transitioning from graduation to novice nurses during the 
COVID-19 pandemic. In addition, nurses' recruitment and employment globally were done rapidly waving many recruitment procedures [9]. In Oman, the nurses' employment was well perceived by the hospitals' administration; however, it is not known how the nurses themselves experienced their first days of employment. What were their motivators? And what were their challenges? Therefore, this qualitative study opened the door for them to share their experiences including their thoughts and opinions to provide support for future newly recruited nurses and to improve the process of employment if required.

\section{Methods}

\subsection{Study Design}

This qualitative study was conducted between May and December 2020 and aimed at describing and comprehending the experiences of the newly employed Omani nurses in the general and critical units at the biggest tertiary hospital in Oman. This study had a qualitative approach and was designed to include semi-structured interviews to identify the nurses' challenges, motivators including their emotional insights for improvement of the future preparatory employment plan.

\subsection{Study Setting}

This study was conducted in the biggest tertiary hospital in Oman, which was commissioned in 1987 in the capital city of Oman and serves as the apex tertiary care referral centre for the country as well as a major teaching hospital. During the pandemic, it was considered a well-structured (COVID-19) centre. The interviews were conducted in a ventilated, quiet, and private room in the hospital to ensure confidentiality. The interviews were conducted at nurses' convenient time with adherence to all protective measures: appropriate physical distance and with protective face masks. All interviews were conducted for $45-60 \mathrm{mi}-$ nutes and digitally recorded.

\subsection{Study Sampling}

Purposive sampling was adopted to include nine newly employed nurses with gender consideration to elicit a variety of experiences among them. In the hospital, the participants were identified as interns who were registered in a three months internship (instead of six months internship due to the (COVID-19) pandemic). Non-Omani interns and who attended more than three months of the internship were excluded from the study.

\subsection{Study Tool}

An interview protocol was designed to guide the interview and elicit more in-depth details. It mirrored the study objectives, and grounded previous research related to novice nurses challenges during an internship with input from a qualitative research expert. The interview protocol was structured by the first 
and the second authors and reviewed by an expert in qualitative research (the fifth author). The interview protocol composed of demographic details of the participants, introductory statement, and questions related to participants' experiences, challenges, motivators and insights related to their employment procedures during the pandemic. The protocol also included questions related to the preparatory training that they received during their internship. The protocol was designed in English and Arabic languages. For the validity and reliability to be preserved, continuous clarity-engagement process was adopted. The qualitative research expert (fifth author) was involved to examine the protocol, examine the process of data collection and evaluate the study outcome to be fair, rigorous, and adequate.

\subsection{Data Collection}

The in-charge nurses were informed and briefed about the study and were asked to inform the interns accordingly. The participants were interviewed by three researchers and the interviews were stopped after saturation was reached.

\subsection{Data Analysis}

The interviews were transcribed verbatim and translated when needed. The analysis process was performed through an inductive approach where the codes were identified from data and the entire analysis was performed at the level of manifest qualitative data analysis. Words and sentences containing aspects related to each other were labelled with a code close to the text.

To reinforce the accuracy of transcribed data and analysis, the process was supervised by an expert in qualitative research at the hospital and to enhance the credibility of the process, debriefing meetings were conducted with all the researchers and study progress was reported and documented.

\subsection{Ethical Consideration}

The study was approved by The Research and Ethical Review and Approval Committee of the hospital, number SRC\#57/2020. Informed Consent was obtained from all the participants before starting the interviews. All the sounds files and transcriptions were stored in a password-protected computer with personal identities removed. All the participants were informed to have the right to withdraw at any stage of the research project.

\section{Results}

Nine participants (two males and seven females) were interviewed from different specialities. The summary of participants characteristics is shown in Table 1.

The analysis of the interviews with newly employed nurses resulted in capturing their experiences during the COVID-19 pandemic and framed them into four contexts: Introductory Nurses Plan, Hospital Environment, Nurses Characters, and Families \& Relatives. The discussed experiences are related to questions WHAT, WHY, HOW are summarized in Appendix 1. 
Table 1. Participants' characteristics.

\begin{tabular}{ccccc}
\hline Gender & Age (years) & Marital status & Without job since graduation (years) & Permanent residency \\
\hline Female & 27 & Married & 3 & Outside Muscat \\
Female & 26 & Single & 3 & Outside Muscat \\
Male & 24 & Single & 1.5 & Outside Muscat \\
Female & 25 & Single & 2 & Outside Muscat \\
Female & 24 & Single & 1 and 3 months & Muscat \\
Female & 24 & Single & 1 and 3 months & Muscat \\
Male & 23 & Single & 1 year & Muscat \\
Female & 29 & Single & 5 years & Outside Muscat \\
Female & 24 & Married & 1and 3 months & Muscat \\
\hline
\end{tabular}

${ }^{*}$ The recruitment is in the capital city of Oman (Muscat).

The presentation of results follows the order set out in the table and direct quotations from the interviews are included to illustrate how the interpretation is grounded in the data.

\subsection{Introductory Nurses Plan}

\subsubsection{Orientation Programme}

The discussion with newly employed nurses revealed that infection control awareness was mainly given through an online approach which was not the best choice for them and hospital policies and procedures were received as reading materials. Additional physical lectures were supplemented to the interns according to their needs.

"Infection control on-line lectures were not enough for us at that time, ... when we went to the ward we have been told that we are lacking important information, so the in-charge decided to give us additional preparation, she gave us face to face lectures, every day for a full week" (Female, General Ward).

"The clinical nurse educator was available to support and provide us with reading materials such as policy, procedures and protocols" (Male, Emergency Room).

\subsubsection{Internship}

The dialogue between the nurses related to the internship process covered its requirement and duration. In this regard, they disclosed that they are overwhelmed by the tasks with an inability to complete the mandatory courses highlighted by the organization in a short time. They requested to be deployed to the (COVID-19) wards rapidly but the worries of not being ready were clearly stated. Although the internship was shortened by three months due to pandemic circumstances, the period remained intense.

"I am overwhelmed by the internship requirements and unable to finish my mandatory courses" (Female, Critical Care Unit). 
"As an intern, I have no time to read about medications, and the new cases, including completing my mandatory courses. I am worried that I will not be ready and later on after the internship I have to be independent, now I am with the preceptor but later I will be alone" (Female, General Ward).

"The first month was very difficult because we have to full fill certain requirements, you know that we have to finish certain mandatory courses and we don't have time" (Male, Critical Care Unit).

\subsubsection{Preceptors}

According to the interns' discussion, the preceptors' availability and attitude varied according to the preceptorship process. Participants revealed that they were not available daily and not in every circumstance due to some hospital arrangements. Also, they adopted different approaches during the assessments with inconsistency in their levels.

"I was confused as my preceptor was not in the same word" (Female, Critical Care Unit).

"I started in the Intensive Care Unit, it was difficult because every day we had different preceptor" (Male, Intensive Care Unit).

"In the first month they kept us with a preceptor who was going to COVID-19 ICU frequently and I didn't meet her except for three days during that month" (Female, Critical Care).

\subsubsection{COVID-19 Preparations}

The results revealed that the preparatory content provided was stumpy in theoretical knowledge, however, it was provided through tailored departmental training.

"They gave us a mechanical ventilation and $(A B G)$ course, it is important because we have to deal with ventilated patients" (Male, Intensive Care Unit).

"I am willing to go to (COVID-19) ward but not now because I want to learn more and I feel that I am not confident with my theoretical knowledge" (Female, General Ward).

"In regards to (COVID-19), they did not prepare us well, only how to wear $P P E$, but any other information related to (COVID-19) theory, unfortunately not' (Female, General Ward).

\subsection{Hospital Environment}

\section{Senior Staff and "Al-Shifa System"}

The newly employed nurses acknowledged senior nurses' availability with continuous support and encouragement. However, the "Al-Shifa System" which is the patients' information database was described to be new for the interns and very confusing but the challenges were controlled by many training sessions.

"My colleagues in the ward are telling me that work in the pediatric ward is interesting, they motivated me. There is also a continuous encouragement from my seniors and the nurse in charge specifically" (Female, General Ward).

"Sometimes senior nurses ask us if we have any doubts in Al-Shifa System and 
they have provided a dedicated person for Al-Shifa and bedside training to support us" (Male, Emergency Room).

\subsection{Nurses Characters}

\subsubsection{Emotional Insights}

The nurses revealed their emotions as stressed, frightened, worried and happy for different reasons and motivators. For example: being stressed due to lack of (COVID-19) pandemic knowledge and due to many different associated measures around it. On the other hand, they expressed their fear due to their appointment in the hospital and the requirements they have to achieve to reach a high level of standards. Also, they expressed their worries about being not well prepared for self-attainment (accommodation, uniforms), hospital selected wards, English language and their available competencies. Although the negative emotions bothered most of them, happiness was yet described among some of them for being employed after a long time of job seeking, employment at the tertiary hospital with continuous support and encouragement from their senior colleagues during their work.

"We have to wear protective apparatus that made us uncomfortable and difficult to breathe especially through $N 95$ mask" (Female, Medical Ward).

"We received a short notice for the employment, they called us on Thursday to report on Sunday, we could not prepare the uniform" (Male, Intensive Care Unit).

"I am enthusiastic to start working at this hospital, ... I heard that this hospital need highly qualified staff" (Male, Intensive Care Unit).

"I was worried not to be assigned to award that I want, ... I wanted to be in maternity but they did not give me maternity, I do not know if I am prepared for this" (Female, Oncology Ward).

"I stayed at home for one year after my graduation, I am worried about committing mistakes now" (Male, Emergency Room).

\subsubsection{Environment Adaptation}

The nurses revealed that the environment adaptation was performed through two modalities: self-motivation and attendance to colleagues' support through positive thinking, working under pressure, accepting the nature of the job and building confidence together.

"I feel proud of myself, I feel good when someone gives me a positive message that encourages me, I don't regret that I am a nurse, it was my dream since I was a child' (Female, Female Medical2).

"I like the nursing profession since I was a student because dealing with patients and serving them makes me happy, also when patients thank nurses, you feel comfortable" (Male, Emergency Room).

\subsection{Family and Relatives}

\section{Emotional Insights}

Their emotions towards their families and relatives were of mixed nature, they 
included anxious and happy reactions. The anxiety was due to the thinking that there is a chance to catch the virus and bringing it home with a chance to inability to go home daily. On the other hand, happy emotions were described as being a source of income and health information for their families and relatives.

" $M y$ father is always calling me, I did not see him since I started my work, I am missing him" (Female, Female Medical Ward 1).

"My father is happy for me because I stayed for a long time at home, he wanted me to get a job to support him financially" (Female, Female Medical Ward 2).

"My mother is sick for a long time, and my family depends on me to provide them with medical information because I am a nurse" (Female, General Ward).

"My family is stressed, they know from the news that this hospital gets a lot of (COVID-19) patients and they are anxious about us" (Female, General Ward).

\section{Discussion}

This study has explored the experiences of nurses who are recruited in the era of (COVID-19). This study revealed that newly employed nurses encounter significant concerns during their internship and some major concerns are related to the training, information related to (COVID 19), overwhelmed internship requirements and preceptors' level variation. However, the new nurses disclosed that the supportive environment from their immediate supervisors, their colleagues and their motivating factors to continue working in the nursing profession gave them a big success.

In term of the orientation programme provided to the nurses, the study revealed that preparation of the internship programme was available, however it lacks the emphasis on pandemic concepts. Although these nurses were given tailored training at each department, they still felt it was inadequate. This could be related to a lack of the original training programme that they should have received during their studies in the college. This was well emphasized in the literature [10] and declared that the medical team does not have enough knowledge required to deal with pandemics. This deficiency could be an additional factor for being a nurse and working under the stress during the pandemic. This phenomenon is well explained in the literature with a necessity to prepare nurses in advance for critical and emergent healthcare crisis with emphasizing policies and adequate training time [11].

Moreover, the nurses' stress was also related to the overwhelmed orientation programme during the (COVID-19) pandemic, as the programme was shortened from six months duration to three months only with the same mandatory requirements.

In term of preceptors, it was found that due to the pandemic situation, preceptors were not available on daily basis along with their preceptee. This unavailability of preceptor made the interns feel confused, with the inability to gain the maximum internship benefits. Moreover, nurses reported a sense of failure in 
their first month of recruitment due to lack of preparedness and some of them proposed better planning, selection of preceptors especially in extreme cases such as pandemic crisis. This same attitude came in the literature [12], however, some other new nurses tried to adapt to the situation by referring their conditions to their senior nurses at the unit which helped a lot and allowed the new nurses to cope with the stress successfully.

Nurses' stress was significant and was associated with fear towards (COVID-19) infection with lack of knowledge of the virus, and fear of being urgently recruited during the pandemic and appointed at the biggest hospital in the country. Most new nurses were unprepared for the recruitment as it was in the middle of the (COVID-19) era with major lockdown in the country. Moreover, the new nurses felt that they have a sense of inadequacy in knowledge and the English language gave them an additional sense of unpreparedness. The nurses' senses were well supported by the literature [13] and proved that urgent recruitment makes the nurses feel inadequate and unprepared.

This situation was also aggravated by not prepared uniforms, unrecognized transportation and improvised accommodations. The results of this study came to align with other studies in the literature that shows that nurses face stress, anxiety, and social isolation during a pandemic due to different technical or social reasons [10] [11] [14].

Moreover, stress and fear to get infected are well-documented feelings [15]. Therefore, the new nurses were encouraged to report fear of infection or fear of getting ill as the literature stated several adaptation processes [16]. In this study, some nurses reported the same and they referred to their feelings as the "new normal life". This could be due to the maturity and development of the interns during the internship, or due to gaining new experiences related to the precautions and patients recovery. The self-modalities that new nurses used during the internship helped them as well to adapt such as: being a positive thinker, working under pressure, self-motivation and utilizing colleagues support. Some studies referred to the technologies as an adaptation modality and were very well documented [12] [17].

Regardless of all the negative emotions toward the pandemic, nurses still pose a sense of happiness with a positive reaction toward the recruitment. This was evident by their happiness and positive thinking of being recruited during the pandemic and the ability to cope with stress. This is in accordance with previous studies in the literature [18] [19] as the literature in this regard supported our data and mentioned that the ability to adapt to stress, being initiative in the learning process through self-learning \& colleagues support, feeling self-responsible about own development \& patient status and recognizing that pandemic is an opportunity rather than challenge for learning and growth [18].

The literature declared that the majority of nurses report a sense of pride in being at the frontline [10] [14] [18]. In this study, the interns were very committed to their responsibilities and they believed that they should carry on and per- 
form at their maximum level of performance. This was in alignment with the literature that mentioned that nurses have a commitment toward their oath and ready to scarify during a pandemic [16] [18].

This study exposed that new nurses possess some emotions toward their families. These emotions are mixed between fear of infecting the family members and being responsible toward them. The sense of fear was related to the self-awareness that a health care worker could be a carrier of the virus and asymptomatic at the same time and the presence of high-risk individuals at homes such as children, elderly people, and individuals with chronic diseases made this feeling larger.

However, these heavy emotions made the nurses react in different manners such as self-quarantine, physical distancing at home, performing protective measures and becoming less social in comparison to the pre-recruitment period. These protective behaviours are also well described in the literature [10]. Moreover, some nurses reported that they separated themselves from their family especially at the beginning of the pandemic due to the high transmission rate, uncertainties of the disease, and possibility of being an asymptomatic carrier. This could be related to the sense of responsibilities the nurses possess toward their families and desire for maximum family safety and our findings were aligned with the literature as well [16] [20] [21].

In terms of family reaction, all participants expressed that their families had mixed feelings between fear of getting infection and happiness of recruitment. The fear was a normal feeling as the recruitment was during the pandemic and the high possibility that health care workers get the infection. However, a sense of happiness was overcoming the fear as reported by the nurses. This happiness was due to the long waiting time for the job. Therefore, the families gave the new nurses emotional support by continuous motivation, encouragement, and phone calls. Families also tried to adapt and accept the situation (recruitment during a pandemic) by instructing their daughters or sons to apply protective measures, continuous hand hygiene and avoiding any transmission of infection to family members. Regardless of all fear and worries of infection, families were feeling proud of their young nurses being health care workers during the period of the (COVID-19) pandemic.

\section{Methodological Considerations and Limitations}

The study captured a range of experiences related to newly employed nurses. The sample of participants was selected from the newly recruited interns, so the sample served the purpose of the study. The study did not include gender comparison; however, the low number of male participants can be explained by the low numbers of males who graduated from nursing college compared to females. It is not known whether the same results would have been noticed if the gender distribution had been equal.

The data in this study was collected by the first, second and fourth authors, who are Omani clinical nurses and this gave a positive credit for being part of 
the system and having a pre-understanding of the situation. On the other hand, the outsider aspect of the third (administrative nurse) and the fifth (physician) authors gave positive credit for not being judgmental of the challenges and the interns' experiences.

Researchers who conducted this study are from different contexts, and this provides additional positive credits to the study, as what is taken for granted by some researchers might not be well known by others. Regarding data analysis, the first, second and fourth authors performed the first set of coding and the fifth author, who does not work within the system and was considered an external researcher reinforced this with further qualitative data analysis and interpretation. Furthermore, all the authors have different experiences in the field and further discussion was carried out through regular peer debrief sessions.

The study included nine participants. Therefore, the findings might not apply to the other participants. However, the hospital system and the internship programme are almost the same throughout Oman, and nine participants can be considered a representative sample for the newly recruited interns.

\section{Conclusion}

In conclusion, our study showed that newly recruited nurses have a lack of knowledge towards (COVID-19) pandemic which was associated with mixed emotional insights towards themselves, their families and relatives, their preceptors and the hospital environment including their preparatory internship plan. Recruitment during the pandemic has a major impact on nurses' characters, learning process, as well as on their families. However, worries were significantly associated with the time of the pandemic, restriction of social contacts, and the community reaction toward the pandemic situation. Additionally, the preceptorship programme was found to be inconsistent with the novice nurses' needs and was directly related to the redistribution of preceptors to meet the staffing requirements during the pandemic. Nurses' stressors and motivators could be addressed in the future with a clear need for preparatory adjustments to support them with a lot of involvements, shared responsibilities and implications.

\section{Recommendation}

Hospital nursing leaders could play a key role in identifying, prioritizing and preparing nurses for a safe transition into their work environment to mitigate the impact of recruitment in pandemic crisis. The creation of an application form in an online platform could be a resourceful solution providing support for the nurses and could be one of the strategies in preparing nurses to manage their learning process during the internship. The platform could also offer novice nurses a space in which they could share their feelings and experiences and get support from their peers, tutors or senior nurses within the profession. Moreover, an online platform that contains short audios for meditation, short videos or 
reading materials that could aid the nurses in their learning and provide tips for adaptation should be well acknowledged. Along with the use of technology, proper and planned placement of novice nurses with an early prepared preceptor who can offer a learning experience as well as support for the nurse to transition into the workplace should be considered. These measures increase novice nurses' confidence as well as high-quality patient care [12]. Moreover, healthcare institutions and policymakers within the organization need to identify the new novice nurses' stress factors and apply appropriate interventions to maintain their health, safety, and well-being within the working environment [15].

\section{Conflicts of Interest}

The authors declare no conflicts of interest regarding the publication of this paper.

\section{References}

[1] CDC-Centers for Disease Control and Prevention, Coronavirus Disease 2019 (COVID-19) | Disease or Condition of the Week | CDC, CDC, 2020.

https://www.cdc.gov/dotw/covid-19/index.html

[2] Al Wahaibi, A., Al Manji, A., Al Maani, A., Al Rawahi, B., et al. (2020) COVID-19 Epidemic Monitoring after Non-Pharmaceutical Interventions: The Use of Time -Varying Reproduction Number in a Country with a Large Migrant Population. International Journal of Infectious Diseases, 99, 466-472.

https://doi.org/10.1016/j.ijid.2020.08.039

[3] Khamis, F., Al Rashidi, B., Al-Zakwani, I., Al Wahaibi, A.H. and Al Awaidy, S.T. (2020) Epidemiology of COVID-19 Infection in Oman: Analysis of the First 1304 Cases. Oman Medical Journal, 35, e145. https://doi.org/10.5001/omj.2020.60

[4] Khamis, F., Al-Zakwani, I, Al Naamani, H., Al Lawati, S., Pandak, N., et al. (2020) Clinical Characteristics and Outcomes of the First 63 Adult Patients Hospitalized with COVID-19: An Experience from Oman. Journal of Infection and Public Health, 13, 906-913. https://doi.org/10.1016/j.jiph.2020.06.002

[5] Mascha, E.J., Schober, P., Schefold, J.C., Stueber, F. and Luedi, M.M. (2020) Staffing with Disease-Based Epidemiologic Indices May Reduce Shortage of Intensive Care Unit Staff during the COVID-19 Pandemic. Anesthesia and Analgesia, 131, 24-30. https://doi.org/10.1213/ANE.0000000000004849

[6] Murray, M., Sundin, D. and Cope, V. (2018) New Graduate Registered Nurses' Knowledge of Patient Safety and Practice: A Literature Review. Journal of Clinical Nursing, 27, 31-47. https://doi.org/10.1111/jocn.13785

[7] Ortiz, J. (2016) New Graduate Nurses Experiences about Lack of Professional Confidence. Nurse Education in Practice, 19, 19-24. https://doi.org/10.1016/j.nepr.2016.04.001

[8] Norris, H., New, K. and Hinsberg, F. (2019) Patient Deterioration Simulation Education and New Graduate Nurses' Self-Confidence and Competence: A Pilot Study. Journal for Nurses in Professional Development, 35, 330-336. https://doi.org/10.1097/NND.0000000000000588

[9] Stievano, A., Bakhshi, M., Shaffer, F.A. and Hamilton, D. (2021) Ethical Challenges and Nursing Recruitment during COVID-19. Nursing Ethics, 28, 6-8.

https://doi.org/10.1177/0969733021989180 
[10] Kalateh, S.A., Zarei, L., Shahabi, S., Heydari, S.T., Taheri, V., Jiriaei, R., Ebrahimzade, N. and Lankarani, K.B. (2021) Nursing Experiences of COVID-19 Outbreak in Iran: A Qualitative Study. Nursing Open, 8, 72-79. https://doi.org/10.1002/nop2.604

[11] Karimi, Z., Fereidouni, Z., Behnammoghadam, M., Alimohammadi, N., Mousavizadeh, A., et al. (2020) The Lived Experience of Nurses Caring for Patients with COVID-19 in Iran: A Phenomenological Study. Risk Management and Healthcare Policy, 13, 1271-1278. https://doi.org/10.2147/RMHP.S258785

[12] García-Martín, M., Roman, P., Rodriguez-Arrastia, M., Del Mar Diaz-Cortes, M., Soriano-Martin, P.J. and Ropero-Padilla, C. (2020) Novice Nurse's Transitioning to Emergency Nurse during COVID-19 Pandemic: A Qualitative Study. Journal of Nursing Management, 29, 258-267. https://doi.org/10.1111/jonm.13148

[13] Danielis, M., Peressoni, L., Piani, T., Colaetta, T., Mesaglio, M., Mattiussi, E. and Palese, A. (2021) Nurses' Experiences of Being Recruited and Transferred to a New Sub-Intensive Care Unit Devoted to COVID-19 Patients. Journal of Nursing Management, 1-10. https://doi.org/10.1111/jonm.13253

[14] Kim, J. (2017) Nurses Experience of Middle East Respiratory Syndrome Patients Care. Journal of the Korea Academia-Industrial Cooperation Society, 18, 185-196.

[15] Arnetz, J.E., Goetz, C.M., Arnetz, B.B. and Arble, E. (2020) Nurse Reports of Stressful Situations during the COVID-19 Pandemic: Qualitative Analysis of Survey Responses. International Journal of Environmental Research and Public Health, 17, 8126. https://doi.org/10.3390/ijerph17218126

[16] Eftekhar, A.M., Naserbakht, M., Bernstein, C., Alazmani-Noodeh, F., Hakimi, H. and Ranjbar, H. (2020) Healthcare Providers Experience of Working during the COVID-19 Pandemic: A Qualitative Study. American Journal of Infection Control, 49, 547-554. https://doi.org/10.1016/j.ajic.2020.10.001

[17] Al Ghafri, T., Al Ajmi, F., Anwar, H., Al Balushi, L., Al Balushi, Z., et al. (2020) The Experiences and Perceptions of Health-Care Workers During the COVID-19 Pandemic in Muscat, Oman: A Qualitative Study. Journal of Primary Care and Community Health, 11, 1-8. https://doi.org/10.1177/2150132720967514

[18] LoGiudice, J.A. and Bartos, S. (2021) Experiences of Nurses during the COVID-19 Pandemic: A Mixed-Methods Study. AACN Advanced Critical Care, 32, 14-26. https://doi.org/10.4037/aacnacc2021816

[19] Lee, J. and Kang, S.J. (2020) Factors Influencing Nurses' Intention to Care for Patients with Emerging Infectious Diseases: Application of the Theory of Planned Behavior. Nursing and Health Sciences, 22, 82-90. https://doi.org/10.1111/nhs.12652

[20] Lam, K.K. and Hung, S.Y.M. (2013) Perceptions of Emergency Nurses during the Human Swine Influenza Outbreak: A Qualitative Study. International Emergency Nursing, 21, 240-246. https://doi.org/10.1016/j.ienj.2012.08.008

[21] Goulia, P., Mantas, C., Dimitroula, D., Mantis, D. and Hyphantis, T. (2010) General Hospital Staff Worries, Perceived Sufficiency of Information and Associated Psychological Distress during the A/H1N1 Influenza Pandemic. BMC Infectious Diseases, 10, Article No. 322. https://doi.org/10.1186/1471-2334-10-322 


\section{Appendix 1}

\begin{tabular}{ll}
\hline Context & Categories \\
\hline & $\begin{array}{l}\text { 1) Orientation } \\
\text { programme. }\end{array}$
\end{tabular}

\begin{tabular}{ll} 
Sub-Categories & Experiences (WHAT/WHY/HOW) \\
\hline ) Infection control awareness & $\bullet$ On-line approach mainly.
\end{tabular}
a) Infection control awareness.
- On-line approach mainly.
b) Hospital policies and procedures. $\bullet$ Received as reading materials.

a) Requirements.

2) Internship.

b) Duration.

A) Introductory

Nurses Plan.

a) Availability.

3) Preceptors.

b) Attitude.

\section{4) COVID-19}

preparations.

a) Information.

1) Staff. a) Senior Nurses and Leaders

B) Hospital

Environment.

2) Hospital
system.

a) “Al-Shifa” system

a) Stressed.

b) Frightened.

\section{C) Nurses 1) Emotional \\ Characters. insights.}

- Overwhelmed by the tasks.

- Inability to finish the mandatory courses.

- Requested to be deployed to COVID-19 wardshastily.

- Short. (changed from six to three months due to pandemic circumstances).

- Intense.

- Not on a daily bases.

- Rearranging destruction (due toshift assignment between the preceptor and the preceptee).

- Absent in some circumstances.

- Diversebehaviours.

- Different approaches during the assignments.

- Inconsistent in their level.

- Content has stumpytheoretical knowledge.

- Provided through tailored departmental training.

- Available from day one.

- Continuous encouragement.

- Supportive attitude.

- Training received through several sessions.

- New.

- Confusing.

- Catching the virus.

- Lack of COVID-19 virus knowledge.

- Wearing masks and PPE.

- Reduced social contacts.

- Being the source of infection after their family members got infected.

- Not being up to the standard (hospitals need high qualified staff).

- Appointed in words with compromised patients.

- COVID-19 situation.

- Making mistakes as new staff.

- Not being prepared for work due to short notice (uniforms and accommodationsnot prepared).

- Not being prepared to work due to long stay at home after graduation.

- Not to be assigned to a ward of preferred choice.

- Weak English language.

- Being incompetent.

- Recruitment after long timeof job seeking.

- Working at a tertiary hospital.

- Being responsible.

- Senior staff encouragement. (to work independently and ask questions).

- Considering themselvespart of the team.

- Positive thinkersfor being recruited.

- Being passionate about nursing profession.

- Working under pressure.

- Accountable for self-learning.

- Being around patients. (positive patients' feedback).

- Accepting the nature of the job.

b) Attendance to colleagues' support $\bullet$ Building confidence together.

- Working with a sense of competition. 


\section{Continued}

D) Family and 1) Emotional Relatives insights. a) Anxious.

b) Happy.
- Catching the virus.

- Bringing the virus home.

- Separation and inability to go homeon a daily bases due to work requirements.

- For the recruitment.

- Being a source of healthinformationfor their family members

- For supporting their family financially. 